\title{
Development and analysis of a dynamic compartment model to predict carbon-14 behavior in rice paddy field for dose assessment of atmospheric release
}

\author{
By T. Takahashi ${ }^{1, *}$, S. Takahashi ${ }^{1}$, K. Yamamoto ${ }^{2}$, K. Tagami, N. Ishi and S. Uchida \\ ${ }^{1}$ Research Reactor Institute, Kyoto University, 2 Asashiro-nishi, Kumatori-cho, Sennan-gun, Osaka 590-0494, Japan \\ 2 Y First Inc. 937-4, Nishitani-cho, Hodogaya-ku, Yokohama-shi, Kanagawa, 240-0052, Japan National Institute of Radiological Sciences 4-9-1, \\ Anagawa, Inage-ku, Chiba-shi, Chiba, 263-8555, Japan
}

(Received December 11, 2009; accepted in revised form November 12, 2010)

\section{Dynamic compartment model / Carbon-14 / Rice / Atmospheric release / IAEA EMRAS project / \\ Parameter sensitivity analysis}

\begin{abstract}
Summary. Carbon-14 is one of the critical radionuclides for the dose estimation from nuclear fuel reprocessing plants and nuclear waste disposal facilities. To assess the impact on public health caused by carbon-14 released from these nuclear facilities in Japan, a reasonable estimation of internal dose from the rice ingestion is necessary because rice is a staple food. Therefore, we are developing a dynamic compartment model to predict carbon-14 behavior in atmosphere - paddy soil - rice plant system and carbon-14 concentration in rice by using a migration prediction code, MOGRA. In this model, growing curves of "leaf and stem part" and "ear part" of a rice plant were fitted to sigmoid curves. The transfer coefficients between the compartments were estimated by using sub-models and parameters which describe transfer mechanisms of carbon in a rice paddy field, such as photosynthesis, translocation and respiration in a rice plant. A validation study of the model, carried out with "Rice Scenario" presented by the IAEA EMRAS (Environmental Modelling for RAdiation Safety) project, showed that the developed model can be practically used to predict the carbon-14 concentration in rice. The model was also applied for hypothetical releases of carbon-14 from nuclear fuel reprocessing plant. In addition, parameter sensitivity analyses were carried out to select critical parameters.
\end{abstract}

\section{Introduction}

To assess the impact on public health caused by radionuclides released from nuclear facilities by a routine operation or accidental release in Japan, a reasonable estimation of internal dose from the pathway of rice ingestion is necessary because rice is a staple food. Therefore, we are developing dynamic compartment models to estimate the behaviors of some important radionuclides in rice paddy field for safety assessment of nuclear facilities [1-3].

Among the radionuclides of interest, carbon-14 is one of the critical radionuclides for the safety assessment of

\footnotetext{
*Author for correspondence (E-mail: tomoyuki@ rri.kyoto-u.ac.jp).
}

nuclear facilities such as nuclear fuel reprocessing and radioactive waste disposal plants. However, it is difficult to predict carbon-14 behavior with the models which have been developed for other radionuclides, such as radioiodine or radiocesium, because the behavior of carbon in biosphere is much different from these of other elements. Main characteristics of carbon-14 in rice paddy field might be listed as follows;

- $\mathrm{CO}_{2}$ in atmosphere is taken into plant internal and changed to organic matter through photosynthesis,

- organic matter is accumulated in leaf, stem and ear part of rice plant at each growing stage,

- a part of organic boundary carbon is changed to $\mathrm{CO}_{2}$ by respiration, and effused to atmosphere, and

- various chemical forms exist in environmental media such as atmosphere, water and soil.

Therefore, we are developing a dynamic compartment model to predict the carbon-14 behaviors in atmosphere paddy soil - rice plant system and the carbon-14 concentrations in rice grains [3]. In this model, the transfer coefficients between the compartments are estimated by using submodels and parameters which describe transfer mechanisms of carbon in a rice paddy field. A validation study of the model, have been carried out with "Rice Scenario" presented by the IAEA EMRAS (Environmental Modelling for RAdiation Safety) project, showed that the developed model can be practically used to predict carbon-14 concentration in rice.

The model was also applied for hypothetical short term releases of carbon-14 as $\mathrm{CO}_{2}$ from nuclear fuel reprocessing plant. In addition, important parameters which have great influence on the carbon-14 concentration in rice grains at harvest were pick out by parameter sensitivity analyses.

\section{Model description}

\subsection{Construction of dynamic compartment model}

To evaluate the effects of environmental-load substances such as radionuclides, it is essential to identify the behaviors of these substances in the environment. However, the mechanisms involved in the behaviors of substances in the 
environment are very complicated. Therefore, the dynamic compartment model is useful and effective to evaluate the behaviors of substances in the environment [4].

In dynamic compartment models, fluxes of substances among compartments, inflow rate into each compartment and outflow rate from each compartment are usually described as time dependent movement of substances. In such models, simultaneous ordinary differential equations are used to describe the change of inventories of radionuclides in each compartment as follows;

$$
\begin{aligned}
\frac{\mathrm{d} Y_{m, i}}{\mathrm{~d} t}= & -\left(\sum_{l \neq m} k_{m \rightarrow l, i}+\lambda_{m, i}+\lambda_{i}\right) Y_{m, i}+\sum_{l \neq m} k_{l \rightarrow m} Y_{l, i} \\
& +\sum_{j \neq i} p_{j \rightarrow i} \lambda_{j} Y_{m, j}+q_{m, i}
\end{aligned}
$$

where, $Y_{m, i}, Y_{m, j}$ : amount of nuclide $i, j$ in the compartment $m$ (atom), $Y_{l, i}$ : amount of nuclide $i$ in the compartment $l$ (atom), $k_{m \rightarrow l, i}$ : transfer coefficient of nuclide $i$ from the compartment $m$ to the compartment $l\left(\mathrm{~s}^{-1}\right), k_{l \rightarrow m, i}$ : transfer coefficient of nuclide $i$ from the compartment $l$ to the compartment $m\left(\mathrm{~s}^{-1}\right), \lambda_{m, i}$ : elimination coefficient of nuclide $i$ from compartment $m\left(\mathrm{~s}^{-1}\right), \lambda_{i}, \lambda_{j}$ : decay constant of nuclide $i, j\left(\mathrm{~s}^{-1}\right), p_{j \rightarrow i}$ : branching ratio from nuclide $j$ to nuclide $i$ $(-)$, and $q_{m, i}$ : inflow rate of nuclide $i$ into compartment $m$ $\left(\right.$ atom s$\left.~^{-1}\right)$.

A conceptual illustration of the compartment model developed for an atmospheric release of carbon-14 as $\mathrm{CO}_{2}$ from nuclear facilities is presented in Fig. 1 [3]. In this model, photosynthesis, respiration and translocation are considered as the transfer mechanisms of carbon. The organic carbon is produced by photosynthesis and accumulated in leaf, stem and ear parts at each growing stage. After the earing stage, a part of the organic carbon accumulated in leaf and stem parts already is transferred to the ear part. To simplify the model, the carbon-14 released as $\mathrm{CO}_{2}$ from "Leaf and stem organic" compartment and "Ear organic" compartment by respiration assumed to be returned to "Air" compartment directly.

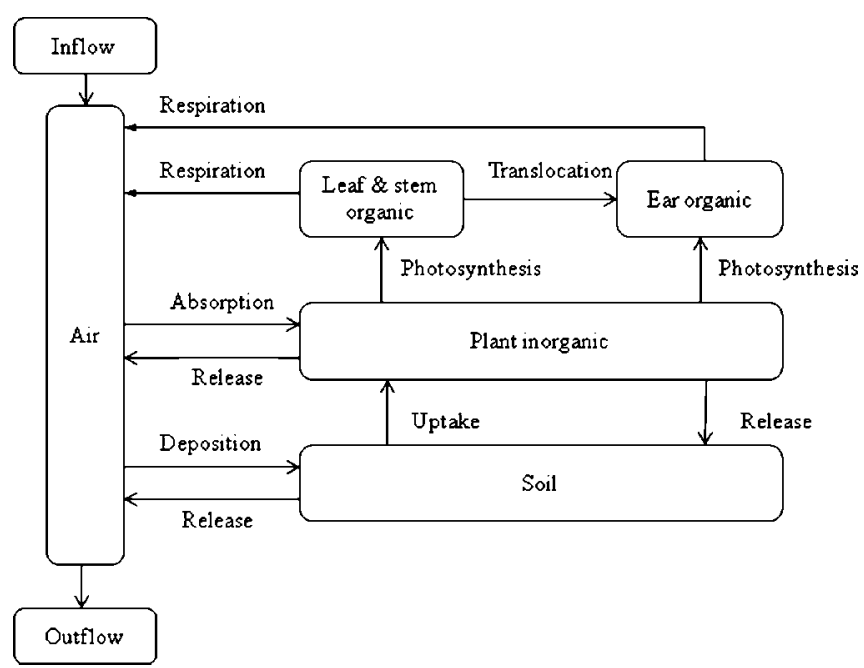

Fig. 1. Conceptual illustration of the compartment model developed for an atmospheric release of carbon-14 as $\mathrm{CO}_{2}$ from nuclear facilities.
In this study, the migration prediction code MOGRA [4] is used for development of the dynamic compartment model and some analyses.

\subsection{Estimation of the values of transfer coefficients}

The transfer coefficients between the compartments and elimination coefficients from the compartments are estimated by using sub-models and parameters which describe transfer mechanisms of carbon-14 in rice paddy field. In this model, the transfer coefficient of each pathway is estimated by considering all the transfer mechanisms in the pathway. For example, the carbon in air is absorbed in a plant as inorganic carbon. The absorbed carbon from air is accumulated in each part of the plant as inorganic or organic carbon, and a part of the organic carbon is released to air by respiration. Therefore, it is necessary to take account of both the increase in carbon in the plant by growing and the replacement of decreasing carbon by the consumption of organic carbon by dark respiration, as the transfer mechanisms from "Air" compartment to "Plant inorganic"compartment, because all of organic carbon in the plant pass through "Plant inorganic" compartment. In addition, exchange of carbon between "Air" compartment and "Plant inorganic" compartment is considered. Photorespiration is not considered as a transfer mechanism because the carbon returns to the air quickly. The transfer coefficient from "Air" compartment to "Plant inorganic" compartment is estimated from all of the following transfer mechanisms;

- increasing of inorganic carbon in rice plant by growing

- increasing of organic carbon in leaf and stem parts by growing,

- increasing of organic carbon in ear part by growing,

- consumption by respiration of organic carbon from leaf and stem parts,

- consumption by respiration of organic carbon from ear part,

- exchange between air and inorganic carbon in rice plant, and

- contribution of carbon in air to total carbon absorbed in a plant.

Growing of rice plants is the most important mechanism to estimate the transfer coefficients. In this study, growing curves of "stem and leaf part" and "ear part" of a rice plant were fitted to sigmoid curves because of simplification of analysis. The sigmoid function used in the model is modified as follows;

$$
\begin{aligned}
& W(t)=W_{\max } \frac{g(t)-g(0)}{g\left(T_{\mathrm{H}}\right)-g(0)} \\
& g(t)=\frac{10^{K\left(t-T_{1 / 2}\right)}}{1+10^{K\left(t-T_{1 / 2}\right)}}
\end{aligned}
$$

where $W(t)$ : weight at time $t\left(\mathrm{~kg}\right.$-dry $\left.\mathrm{m}^{-2}\right), W_{\max }$ : weight at harvest $\left(\mathrm{kg}\right.$-dry $\left.\mathrm{m}^{-2}\right), t$ : time from transplanting $(\mathrm{d}), T_{\mathrm{H}}$ : time at harvest (d), $K$ : constant $\left(\mathrm{d}^{-1}\right)$, and $T_{1 / 2}$ : time when weight is half of harvest weight (d).

The parameters used in this function are estimated from measured values [5]. The parameter values used in this model are shown in Table 1 and the estimated growing 


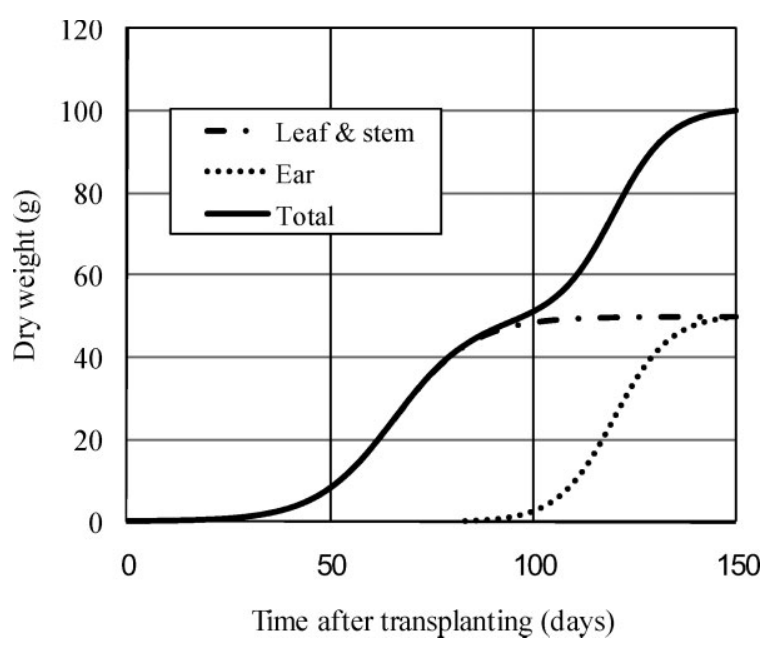

Fig. 2. Growing curves of each part of rice plant.

\begin{tabular}{lccc}
\hline Parameter & Unit & Leaf \& stem parts & Ear part \\
\hline$K$ & $\mathrm{~d}^{-1}$ & 0.045 & 0.063 \\
$T_{1 / 2}$ & $\mathrm{~d}$ & 66 & 120 \\
$W_{\max }(t)$ & $\mathrm{g}$ & 50 & 50 \\
\hline
\end{tabular}

Table 1. The parameter values used for growing curves.

curves are shown in Fig. 2. It is assumed that the date of transplanting of a seedling is the 19 May and a growing period is 150 days.

As shown in Fig. 1, two pathways are considered for accumulation of carbon in "Ear organic" compartment. One is a transfer pathway from the "Plant inorganic" compartment. This pathway means the direct accumulation of photosynthetic products at the growing stage. Another is a transfer pathway from "Leaf \& stem organic" compartment. This indicates the removal of organic carbon which was accumulated in leaf and stem parts before then. The function of the ratio of each pathway is estimated from the study on the time dependency distribution ratio of photosynthetic products in a rice plant [6] as follows;

$$
\gamma(t)=1-r_{\text {rem }} \frac{\mathrm{d} W_{\mathrm{E}}(t)}{\mathrm{d} t} /\left(\frac{\mathrm{d} W_{\mathrm{E}}}{\mathrm{d} t}\right)_{\text {max }}
$$

where, $\gamma(t)$ : ratio of direct accumulation of photosynthetic products to total accumulation in ear part at time $t(-), r_{\text {rem }}$ : constant on removal of organic carbon which was accumulated in leaf and stem parts $(-), W_{\mathrm{E}}(t)$ : weight of ear part at time $t(\mathrm{~kg})$, and $\left(\frac{\mathrm{d} W_{E}}{\mathrm{~d} t}\right)_{\max }: \frac{\mathrm{d} W_{\mathrm{E}}(t)}{\mathrm{d} t}$ at $t=T_{1 / 2}$ of ear.

It is estimated that the value of $r_{\text {rem }}$ is 0.7 from experimental result [6].

In this study, both of growth respiration and maintenance respiration are considered for the consumption of organic carbon. For example, the transfer coefficient concerned in respiration of organic carbon in ear part is defined as follows [7];

$$
k_{\text {AP_Eres }}(t)=\left(k_{\mathrm{E}} \frac{\mathrm{d} W_{\mathrm{E}}(t)}{\mathrm{d} t}+c_{\mathrm{E}} \alpha_{\mathrm{E} \_ \text {org }} W_{\mathrm{E}}(t)\right) / \alpha_{\mathrm{E} \_ \text {org }} W_{\mathrm{E}}(t)
$$

where, $k_{\mathrm{AP} \_ \text {Eres }}(t)$ : transfer coefficient due to consumption by respiration from "Ear organic" compartment at time $t\left(\mathrm{~d}^{-1}\right)$,
$k_{\mathrm{E}}$ : constant on growth respiration from ear part $(-), c_{\mathrm{E}}$ : constant on maintenance respiration from ear part $\left(\mathrm{d}^{-1}\right)$, and $\alpha_{\text {E_org }}$ : weight ratio of organic carbon in ear part (-).

The similar equation is used for transfer coefficient due to consumption by respiration from "Leaf \& stem organic" compartment. The values of constants on respiration are estimated from the study on the respiration of rice plant [6-8]. The estimated values of constants on growth respiration from "leaf and stem parts" and "ear" part are 0.25 and 0.15 , respectively. These on maintenance respiration from each part are $0.01 \mathrm{~d}^{-1}$.

\section{Model validation}

\subsection{Analysis for continuous atmospheric release}

To verify the model, the analysis for hypothetical continuous atmospheric release of carbon-14 was carried out. The inflow rate of carbon-14 as $\mathrm{CO}_{2}$ into "Air" compartment was assumed to be constant (3000 Bq per day) and the volume of "Air" compartment $100000 \mathrm{~m}^{3}$. As shown in Fig. 3, the estimated concentration of carbon-14 in each compartment became in equilibrium, rapidly. In equilibrium condition, there was no difference among the concentrations in every compartment. These results are the same as the results obtained by using the specific activity method. This result indicated that the functions of dynamic compartment model developed in this study are verified.

\subsection{Application to the "Rice Scenario" given by IAEA EMRAS project}

For a validation of the model, we applied the developed model to the "Rice Scenario" given by IAEA EMRAS project [9]. In this scenario, some monitoring data, such as weekly carbon-14 discharge rates from the stacks of reprocessing plant at Tokai-mura in Japan and meteorological data observed at the site were given. The calculation end points of "Rice Scenario" were concentrations of carbon-14 in air and rice grains at some points near the reprocessing plant. We applied the developed model and parameters for the estimation of the concentration in rice grains. In this

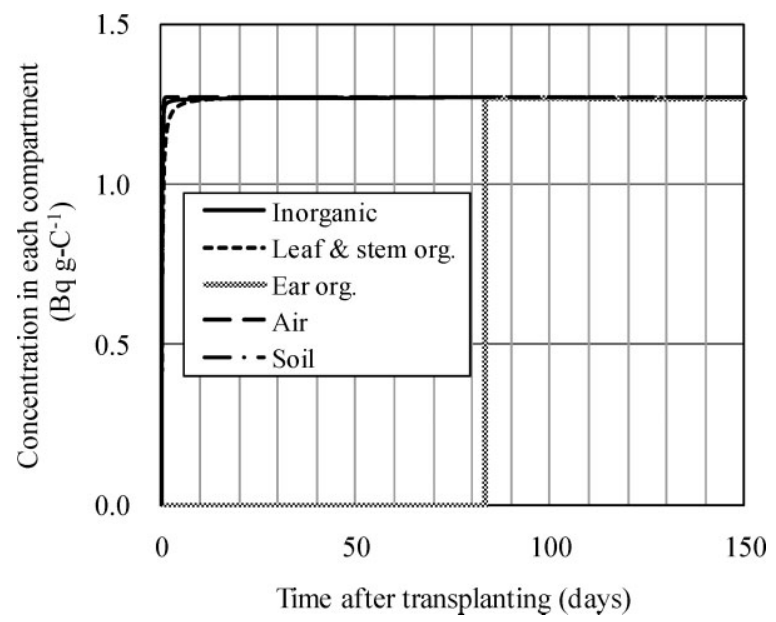

Fig. 3. Estimated concentrations of carbon-14 in each compartment for continuous atmospheric release. 


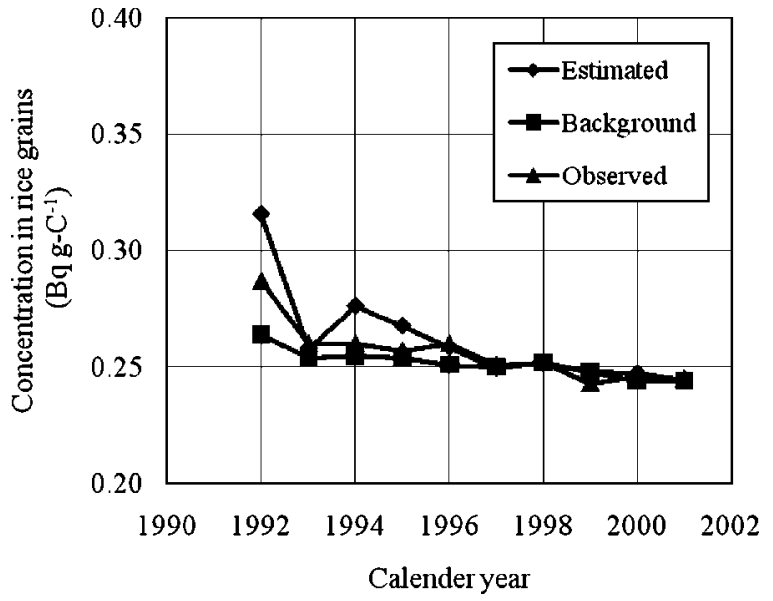

Fig. 4. Results of estimated carbon-14 concentrations in rice grains at monitoring point $\mathrm{R}-1$.

analysis, the inflow rates from the source term to "Air" compartment were calculated by concentrations of carbon-14 in air at each point which were estimated by using Gaussian plume model. For example, the results of estimated carbon14 concentrations in rice grains at monitoring point R-1 are shown in Fig. 4. The estimated results agreed well with the observed data. This result indicated that the developed model and estimated parameters in this study are valid.

\section{Analyses and discussion}

In order to assess the internal radiation dose from carbon-14 after ingestion with rice, it is important to make clear how the timing of carbon-14 release from nuclear facilities affects their concentrations in rice grains at harvest. Therefore we applied the model for hypothetical short term releases of carbon-14 as $\mathrm{CO}_{2}$ from nuclear fuel reprocessing plant, and estimated the contribution ratio of each month in growing period. The estimated contribution ratios of each month for "Leaf \& stem organic" compartment and "Ear organic" compartment at harvest are shown in Fig. 5. The release in August is most effective to both concentration of carbon-14 in "Leaf \& stem organic" and "Ear organic" compartments at harvest, indicating that the release at earing stage is most important to accumulation of carbon-14 in rice plant and rice grains at harvest.

In this study, some parameter values, which concern with growing of rice plant, photosynthesis and respiration, were estimated from previous studies [5-8]. However, these values are changeable by growing conditions such as meteorological condition in growing period. Therefore, it is necessary to select the critical parameters which have great influence on the carbon-14 concentration in rice grains at harvest. The sensitivity analyses of these parameters were carried out to select critical parameters for carbon-14 atmospheric releases.

The function of the ratio of direct accumulation of photosynthetic products to total accumulation of organic carbon in ear part at the growing stage is given by Eq. (3). In the equation, $r_{\text {rem }}$ value was estimated from experimental results, but may be possible to change widely. Therefore, we carried out a sensitivity analysis of $r_{\text {rem }}$ to carbon-14 concentration

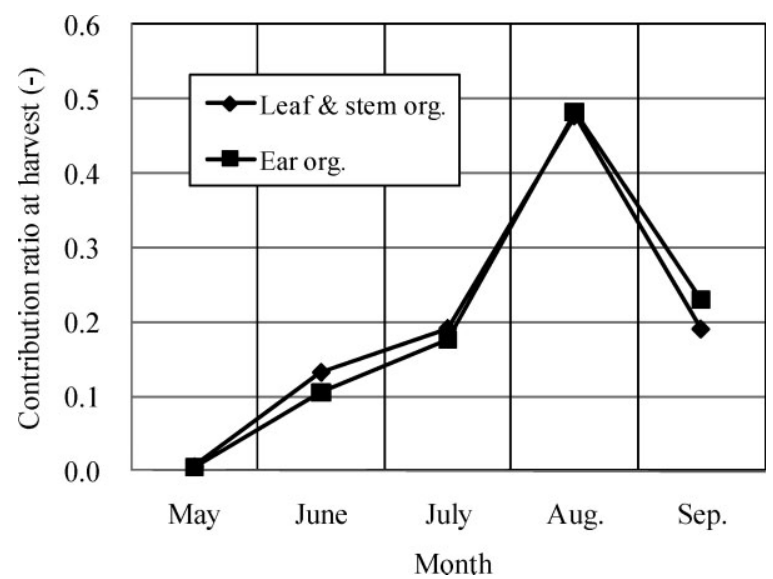

Fig. 5. Estimated contribution ratios of each month for "Leaf \& stem organic" compartment and "Ear organic" compartment at harvest.

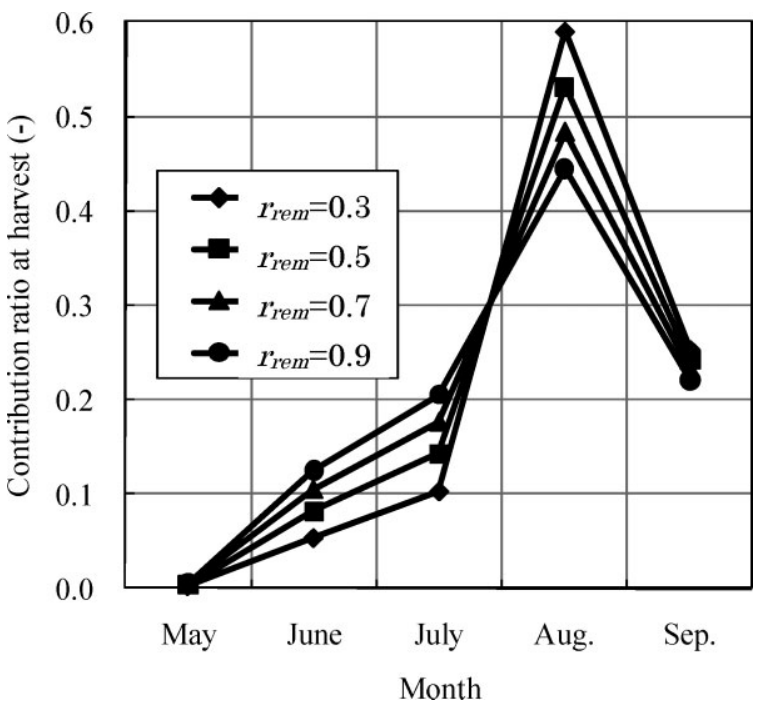

Fig. 6. Relationship between $r_{\text {rem }}$ value and contribution ratios of each month in ear part at harvest.

in rice grains at harvest. As shown in Fig. 6, increase in the $r_{\text {rem }}$ value decreased in a contribution ratio of August and increased in contribution ratios of June and July. The contribution ratios of May and September were almost constant. These results suggest that the ratio of direct accumulation of photosynthetic products to total accumulation of organic carbon in ear part at the growing stage have a great influence on the contributions at tillering stage, reproductive stage and earing stage to the carbon- 14 concentration in rice grains at harvest.

The transfer coefficient due to consumption by respiration is given by Eq. (4). It is known that the values of the constants used in the equation depend on some growing conditions such as growing stage, mutual shielding and so on $[7,8]$. Therefore, we carried out a sensitivity analysis of these constants on growth respiration and maintenance respiration to carbon-14 concentration in rice grains at harvest. The results are shown in Figs. 7 and 8. Fig. 7 indicated that the constants on growth respiration of both of "leaf \& stem" parts and "ear" part had little influence on the contribution ratios of every month, because both of accumulation of organic carbon by photosynthesis and consumption of organic 


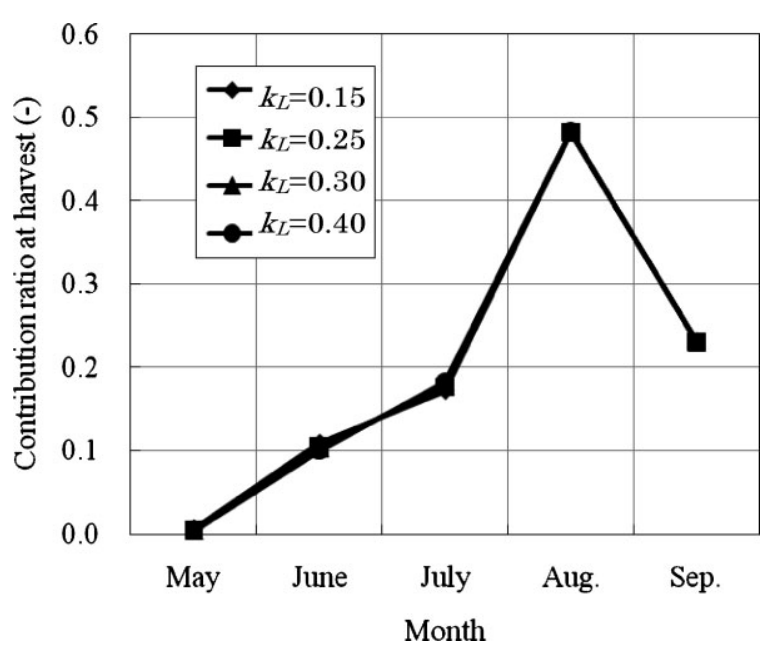

Fig. 7. (a) Relationship between constant on growth respiration of leaf and stem parts and contribution ratios of each month in ear part at harvest.

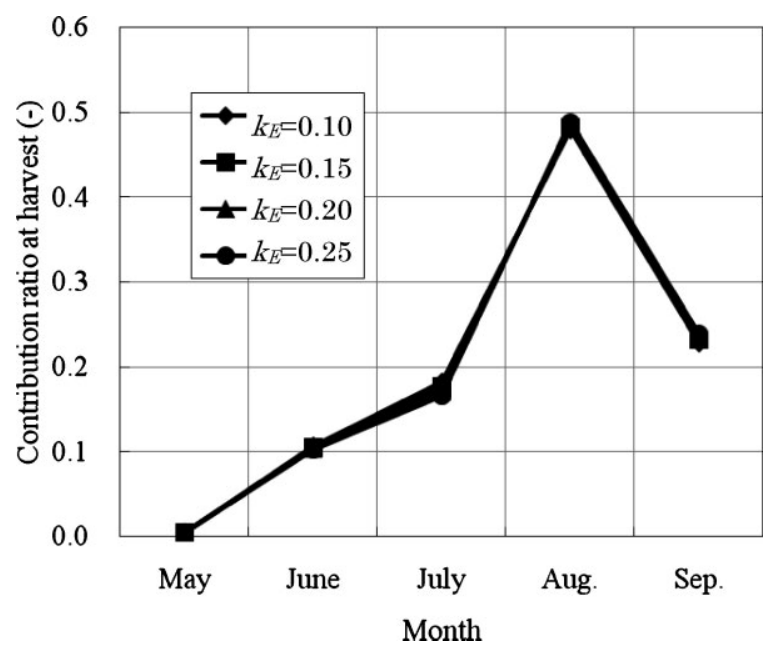

Fig. 7. (b) Relationship between constant on growth respiration of ear part and contribution ratios of each month in ear part at harvest.

carbon by growth respiration are proportioned to differential value of growing curve.

On the other hand, the constants on maintenance respiration were effective to the contribution ratios (Fig. 8). The constant of "leaf \& stem" parts had an influence on the contribution ratios of June. This result suggests that maintenance respiration of leaf $\&$ stem has an influence on the contributions at tillering stage, because decreasing rate of organic carbon-14 accumulated in leaf and stem is affected by the maintenance respiration. In addition, the constant of "ear" part had also a great influence on the contribution ratios of September, suggesting that maintenance respiration of ear has a great influence on the contribution at ripening stage. That is why the organic carbon-14 accumulated in ear is replaced by photosynthetic products until harvest with the maintenance respiration of ear.

\section{Conclusion}

We developed a dynamic compartment model to predict carbon-14 behavior in rice plants and its concentration in

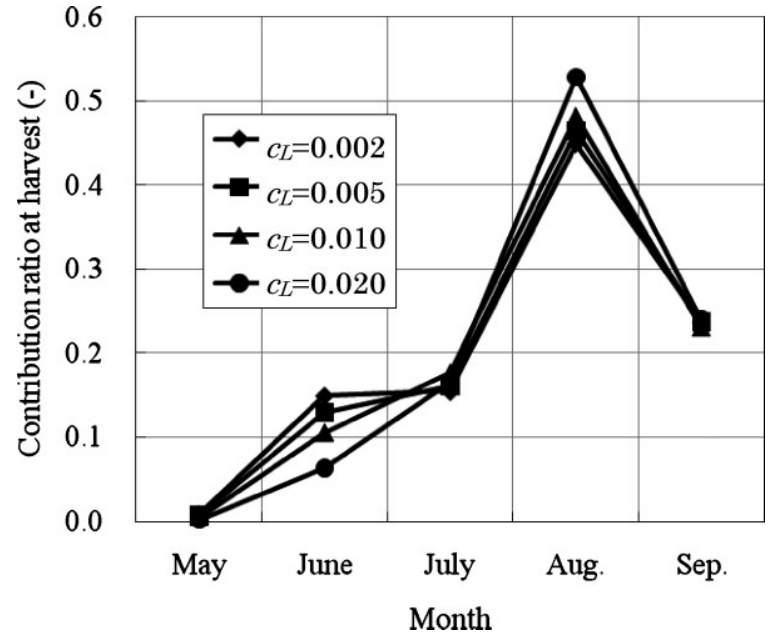

Fig. 8. (a) Relationship between constant on maintenance respiration of leaf and stem parts and contribution ratios of each month in ear part at harvest.

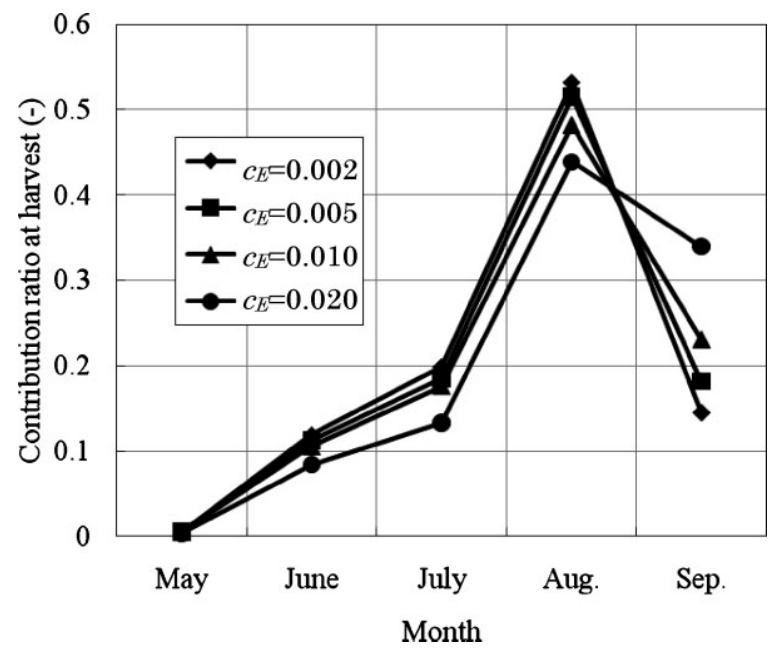

Fig. 8. (b) Relationship between constant on maintenance respiration of ear part and contribution ratios of each month in ear part at harvest.

rice grains. We estimate the transfer coefficients between the compartments by using more appropriate sub-models and parameters which describe the transfer mechanisms of carbon-14 in a rice paddy field. A validation study of the model was carried out with "Rice Scenario" presented by the IAEA EMRAS project.

The model was applied for hypothetical short term releases of carbon-14 as $\mathrm{CO}_{2}$ from nuclear fuel reprocessing plant. In addition, we carried out parameter sensitivity analyses. Important findings in this study are as follows:

- The release of carbon-14 at earing stage is most effective on the accumulation of carbon-14 in rice plant and rice grains at harvest.

- The ratio of the direct accumulation of photosynthetic products to the total accumulation of organic carbon in ear part at the growing stage have a great influence on the contributions at tillering stage, reproductive stage and earing stage to the carbon-14 concentration in rice grains at harvest

- Growth respiration has little influence on the contribution ratios of every month. 
- Maintenance respiration of ear has a great influence on the contribution at ripening stage.

In the future, it is expected that more applicable sub-models and parameter values are estimated by experimental study and/or environmental monitoring data. In addition, it is very important to develop a carbon-14 migration model for wet source terms such as irrigation water for an assessment of waste disposal facilities.

Acknowledgment. This work was partially supported by the Agency for Natural Resources and Energy, the Ministry of Economy, Trade and Industry (METI), Japan.

\section{References}

1. Takahashi, T., Uchida, S., Hayashi, H., Matsuoka, S., Tomita, K., Kurosawa, N.: Development of dynamic compartment model for prediction of behavior of radioiodine in rice paddy field. J. Radioanal. Nucl. Chem. 272, 451 (2007).

2. Takahashi, T., Tomita, K., Yamamoto, K., Uchida, S.: Development of dynamic compartment model for prediction of radionuclide behaviors in rice paddy fields. International Symposium on Environmental Modeling and Radioecology, Institute of Environmental Sciences (2007), p. 189.
3. Takahashi, T., Yamamoto, K., Tagami, K., Takeda, H., Uchida, S.: Development of a dynamic compartment model for prediction of transfer of carbon-14 to rice grains from the atmosphere. Proceeding of the International Symposium on Application of a Closed Experimental System to Modeling for ${ }^{14} \mathrm{C}$ Transfer in the Environment, Institute for Environmental Sciences (2008), p. 15.

4. Amano, H., Takahashi, T., Uchida, S., Matsuoka, S., Ikeda, H., Hayashi, H., Kurosawa, N.: Development of a code MOGRA for predicting the migration of ground additions and its application to various land utilization areas. J. Nucl. Sci. Technol. 40, 975 (2003).

5. Ishizuka, Y., Tanaka, A.: Studies on rice growing no. 1 uptake of inorganic nutrition. Jpn. J. Sci. Plant Nutr. 23, 23 (1953) [in Japanese].

6. Ohsaki, M., Tanaka, A.: Stock material and initial photosynthetic products as the respiration substrate in rice. Jpn. J. Sci. Plant Nutr. 50, 540 (1979) [in Japanese].

7. Ohsaki, M., Tanaka, A.: Relationship between "stock material respiration and initial photosynthetic products" and "growth respiration and maintenance" in rice. Jpn. J. Sci. Plant Nutr. 53, 93 (1982) [in Japanese].

8. Amthor, J. S.: Respiration and Crop Productivity. Gakkai Shuppan Center, Tokyo (2001), p. 140 [in Japanese, translated by Shinano, T.].

9. IAEA: Activities of the EMRAS Working Group on the Modelling of Tritium and Carbon-14 Transfer to Biota and Man, http://www-ns.iaea.org/projects/emras/draft-reports.htm, accessed 27 November 2009. 